\title{
Integration durch nachbarschaftliche Hilfe und soziale Vernetzung
}

Fünf Jahre Modellprojekt »Familie im Stadtteil «

\author{
Gerd Gehrmann, Klaus D. Müller, Uwe Säuberlich
}

\begin{abstract}
Das Programm »Familie Im Stadtteil « in Bremerhaven ist ein Hilfeangebot für Familien mit einem oder mehreren kleinen Kindern, die keine oder nur ungenügende Unterstützung durch ibre eigene Familie, Verwandte, Nachbarn oder Freunde haben. Ein Ziel ist es, Stress und Überforderung von Eltern vorzubeugen und so auch zur Gewaltprävention beizutragen.
\end{abstract}

Das Modellkonzept »Familie im Stadtteil « (FiS) wurde erstmals im Jahre 2000 veröffentlicht. (1) Ende 2005 wurde das theoretische Konzept mit Mitteln der Europäischen Kommission und der Stadt Bremerhaven von der Initiative Jugendhilfe Bremerhaven (2) modellgetreu umgesetzt. Das Methodenhandbuch zum Programm wurde 2008 veröffentlicht. (3)

Die Anschubförderung der Europäischen Union schloss eine wissenschaftliche Begleitung durch die Fachhochschule Frankfurt am Main und eine Beratung durch Experten aus Linz, Amsterdam, Stettin und Wetzlar ein. Diese Förderung wirkte nachhaltig: Zum einen entstand aus der Expertengruppe von Jugendhilfepraktikern und Hochschullehrern das Europäische Netzwerk Gewaltprävention (E.N.G.), dessen Beteiligte sich jährlich zweimal treffen, um Erfahrungen auszutauschen und Projekte zu initiieren, so beispielsweise ein transnationaler Praktikeraustausch seit 2009. Seit 2007 wird das Projekt ausschließlich mit Mitteln der Stadt Bremerhaven finanziert: Das sind drei volle Teamerstellen und laufende Betriebskosten für ein präventives Projekt, dessen positive Wirkungen erst mittelund langfristig nachweisbar werden. Unsere Überlegungen bei der Einführung von »Familie im Stadtteil « waren:

- Hilfen, die Stress bei Eltern abbauen, können Gewalt gegenüber Kindern verhindern.

- Präventive Hilfe ist billiger als Krisenintervention.

- Professionelle Leitung und Organisation aktiviert Potentiale freiwilliger Arbeit.
- Nachbarschaftliche soziale Vernetzung und gegenseitige Hilfe verhindert und beendet die Isolation von Familien.

- Soziale Netzwerkentwicklung ist effektives Quartiersmanagement und wirkt der Bildung von Slums entgegen.

- Soziale Vernetzung und multiethnische Selbsthilfe fördert die Integration ethnischer Minderheiten und Migranten.

\section{Wie man Gewalt gegen Kinder vorbeugen kann}

»Familie im Stadtteil « ist ein präventives Programm. Es soll Stress bei jungen Familien, insbesondere bei Familien mit nur einem Elternteil vermeiden helfen, weil Stress oft die Voraussetzung für Gewalt gegen Kinder ist.

Für die Förderung und Finanzierung von »Familie im Stadtteil « gebührt der Stadt Bremerhaven große Anerkennung. Bei einem präventiven und niederschwelligen Angebot kann die Gewalt vermeidende Wirkung nicht kurzfristig und nicht einfach nachgewiesen werden. Insofern gleicht die Einführung von »Familie im Stadtteil « durch die Stadt einem Sprung ins kalte Wasser. Aus der Literatur und von der Praxis anderer Programme (4) wissen wir jedoch, dass überlastete und gestresste Eltern leicht in Situationen geraten, in denen sie Aggressionen gegenüber ihren Kindern entwickeln und diese verbal und nonverbal (»Klaps«) ausdrücken.

Hierzu ein Beispiel: Jeder hat im Supermarkt schon eine junge Mutter mit kleinen Kindern erlebt. Die Kinder sind bei einem längeren Einkauf gelangweilt, vor allem in der Schlange vor der Kasse. Sie wollen dies und das haben, was die Mutter nicht kaufen will oder kann. Sie laufen durch die Gänge und fassen alle möglichen Artikel und auch Esswaren an. Falls die Mutter hier Einhalt gebietet, tut sie das gestresst. Sie wird laut, schreit die Kinder an und gibt ihnen einen Klaps. 
Das allein ist noch keine Misshandlung, könnte jedoch der erste Schritt dahin sein.

Um diese Situation zu vermeiden, wäre Hilfe für die Mutter wichtig. Es könnte beispielsweise jemand mit den Kindern spielen, damit die Mutter in Ruhe einkaufen gehen kann. Alleinstehende Mütter haben jedoch oft niemand, den sie darum bitten können. Familienangehörige sind nicht da oder anderweit beschäftigt und Nachbarinnen oder die »Oma « von nebenan nicht vorhanden.

Solche geringen Aggressionshandlungen drücken den Stress aus, dem Mütter ausgesetzt sind. Wenn sich Stress und Belastungen anhäufen, können sie dazu führen, dass Kinder geschlagen werden. Um diesen ersten Schritten zur Gewalt gegenüber Kindern vorzubeugen, sollen mit der Hilfe von »Familie im Stadtteil « Situationen vermieden werden, in denen genervte Eltern sich aggressiv gegenüber ihren Kindern verhalten.

Deshalb organisiert »Familie im Stadtteil « nachbarschaftlichen Hilfen zur Stressvermeidung und zur sozialen Vernetzung junger Familien systematisch, professionell und semiprofessionell auf nachbarschaftlicher Ebene. "Familie im Stadtteil « ist von jeder anderen Hilfe für Familien abgegrenzt. Sie ist keine billige Familienhaushaltshilfe und schon gar keine Sozialpädagogische Familienhilfe (SPFH). Die Unterstützungsleistungen sind einfacher Art, erfolgen kurzfristig und vorübergehend. Unsere Überlegungen bei der Einführung von »Familie im Stadtteil« waren:

- Präventive Hilfe erspart jungen Eltern und Kindern vermeidbares Leid, das aufgrund von Unwissenheit, Hilflosigkeit und fehlenden Kompetenzen entsteht, wenn beispielsweise Schläge als Erziehungsmittel eingesetzt werden.

- Präventive Hilfe und Unterstützung erspart alle möglichen späteren Maßnahmen der Krisenintervention, die teurer sind als »Familie im Stadtteil «.

- Prävention erspart jungen Eltern und ihren Kindern auch spätere Deprivierung und Deklassierung.

- »Familie im Stadtteil « kann der Isolation und Ghettoisierung junger Familien, insbesondere von Migranten und ethnischen Minderheiten entgegenwirken, indem das Programm den Aufbau eines informellen sozialen Netzwerks nachbarschaftlicher Hilfen und Freundschaften fördert. Ein solches soziales Netz ergänzt das formelle, verhindert eher Passivität und kann Alltagsdingen effektiver unterstützen kann als jede Agentur.

- »Familie im Stadtteil « wirkt durch Vernetzung der Verslummung von Wohnquartieren entgegen.

- »Familie im Stadtteil « fördert nachweislich die Integration ethnischer Minderheiten und kann damit gegen die Bildung von "Parallelgesellschaften «, Rassismus und Fremdenfeindlichkeit wirken.

\section{Wie »Familie im Stadtteil « in der Praxis funktioniert}

Vor fünf Jahren hat »Familie im Stadtteil « in Bremerhaven-Mitte und Bremerhaven-Nord mit der Arbeit angefangen. Inzwischen wurde »Familie im Stadtteil «

\begin{tabular}{|c|c|c|}
\hline $\begin{array}{l}\text { Das Leben sinnvoll füllen, sich weiter entwickeln } \\
\text { Beispiele für Aussagen: } \\
\text { - "... möchte das Leben mit Inhalten füllen, es stärkt mich, mitmachen zu können ... « } \\
\text { - "Die Arbeit im sozialen Bereich füllt mich aus, schafft Zufriedenheit. « } \\
\text { - »Die Arbeit mit Kindern und Familien bereitet mir viel Spaß.« } \\
\text { - »Ich möchte raus aus dem Haus, eine sinnvolle Aufgabe haben.« } \\
\text { - "Arbeit im Sozialbereich als Alternative zum gelernten Beruf. « }\end{array}$ & 43 Nennungen & 69 Prozent \\
\hline $\begin{array}{l}\text { Eigene Erfahrungen und Kenntnisse weitergeben } \\
\text { Beispiel für Aussagen: } \\
\text { »Ich denke, dass ich in diesem Bereich viel Erfahrungen gemacht habe und diese weiter- } \\
\text { geben kann. " }\end{array}$ & 38 Nennungen & 61 Prozent \\
\hline $\begin{array}{l}\text { Helfen aus eigener Betroffenheit und aus Solidarität } \\
\text { Beispiele für Aussagen: } \\
\text { - "... hätte früher auch gerne Hilfe gehabt. « } \\
\text { - »Ich möchte Familien helfen, weil es anderen viel schlechter geht als mir und ich viel zu } \\
\text { geben habe. « } \\
\text { - »Ich weiß, dass man auch mal Zeit für sich braucht. Ich habe selbst vier Kinder, die } \\
\text { schon erwachsen sind." }\end{array}$ & 35 Nennungen & 56 Prozent \\
\hline $\begin{array}{l}\text { Kontakte zu anderen Menschen haben } \\
\text { Beispiel für Aussagen: } \\
\text { »Ich möchte andere Menschen kennenlernen. « }\end{array}$ & 32 Nennungen & 52 Prozent \\
\hline $\begin{array}{l}\text { Interesse an anderen Kulturen und Lebensstilen } \\
\text { Beispiel für Aussagen.: } \\
\text { »Ich möchte andere Kulturen kennenlernen. « }\end{array}$ & 15 Nennungen & 24 Prozent \\
\hline
\end{tabular}

Tabelle 1: Die Motive der Freiwilligen im Programm »Familie im Stadtteil « entsprechen dem Typus »Neues Ehrenamt. (Die Prozentzahlen addieren nicht auf 100, weil Mehrfachnennungen möglich waren.) 
auch auf Bremerhaven Süd und damit auf ganz Bremerhaven ausgeweitet.

Es war ein großer strategischer Vorteil, dass das erste Büro von »Familie im Stadtteil « als Anlaufstelle in einer großen, zentral gelegenen Kindertagesstätte der Initiative Jugendhilfe Bremerhaven eingerichtet wurde, deren Leiterin eine Ausbildung zur Teamleiterin von »Familie im Stadtteil « erfolgreich abgeschlossen hat. Sie kannte also das Programm gut und sah in »Familie im Stadtteil « ein sinnvolles Hilfeangebot für die Eltern der Kinder in der Kita. (5)

Durch Flyer und die Lokalpresse wurden das Programm und sein Angebot in der Stadt veröffentlicht. Gleichzeitig mit dem Angebot an Eltern wurden Freiwillige für die Ausbildung und Arbeit als Assistentin in »Familie im Stadtteil « angeworben. Skeptiker bezweifelten, ob es genug Freiwillige für die Arbeit geben würde. Die Realität hat jedoch unsere Erwartungen übertroffen. Es gibt offensichtlich ein großes Potential an - überwiegend - Frauen, die bereit sind, sich für eine vernünftige Sache zu vernünftigen Bedingungen zu engagieren.

Ende 2009 waren bei »Familie im Stadtteil« 63 Frauen und zwei Männer als Assistenten in "Familie im Stadtteil « (FiS) aktiv, darunter etwa die Hälfte Frauen mit Migrationshintergrund, mehr als ein Drittel aller FiS-Assistenten sind Moslems. Die Zahl der Bewerberinnen war größer, nicht alle waren geeignet.

Die Frauen entsprechen dem neuen Typus des Ehrenamts: Sie suchen eine sinnvolle Betätigung im sozialen Bereich, die nach Umfang und Dauer klar umrissen ist, sie schätzen die Ausbildung und Aufmerksamkeit der Teamleiterinnen, die Kontakte mit den Familien und anderen Kolleginnen, freuen sich über eine geringe finanzielle Anerkennung und fühlen sich dadurch geehrt, dass sie den akzeptierten Titel »FIS-Assistentin « tragen dürfen.

Besonders wichtig ist den Freiwilligen, dass sie sich im Engagement für andere selbst verwirklichen können. Ihren Dank uns Initiatoren von »Familie im Stadtteil « gegenüber, die ihnen ihr soziales Engagement ermöglichten, haben die FiS-Assistentinnen in einer Veranstaltung gezeigt, die sie für uns im Herbst 2009 organisierten. Treibende Kraft waren dabei moslemische Frauen. Türkische und arabische FiS-Assistentinnen arbeiten als Helferinnen, weil es auch eine Möglichkeit bietet, aus der Isolation herauszukommen, Sprachkenntnisse zu erweitern und gesellschaftliche Anerkennung zu erhalten und können dabei ihren kulturellen Hintergrund für ihre Arbeit nutzen.

Die Anwerbung, Ausbildung und Anleitung der FiS-Assistentinnen ist Aufgabe der hauptamtlichen FiS-Teamleiterinnen: vier Frauen und ein Mann auf einer vollen und vier halben Stellen. Die Teamleiterin mit der vollen Stelle fungiert als Gruppenleiterin und Koordinatorin.

Es war also nicht schwer, genug freiwillige Helferinnen anzuwerben, was mit ihrem Bedürfnis nach sozialer Betätigung außerhalb von, aber verbunden mit ihrer eigenen Familie zusammenhängt. Die Motive für ihr Engagement entsprechen dem Typus "Neues Ehrenamt « (vgl. Tabelle 1). Es sind - wie deutlich wird nicht die klassischen Helfermotive, die hier überwiegen. Dominierend erscheint das Motiv, das Leben sinnvoll zu füllen, etwas Neues zu tun und sich weiter zu entwickeln. In dem sie anderen helfen, tun sie etwas für sich.

FiS-Assistentinnen wollen auch eigene Kenntnisse weitergeben. Auch das Hilfemotiv spaltet sich noch einmal auf: Zwölf FiS-Assistentinnen engagieren sich, weil sie selbst schon mal in der Lage von FiSFamilien waren. Ein anderes starkes Mo-

\begin{tabular}{|l|l|l|}
\hline Mutter und Vater mit Kindern & 82 Fälle & 72,5 Prozent \\
\hline Alleinerziehende Mütter & 23 Fälle & 20,5 Prozent \\
\hline Mütter mit Partnern & 8 Fälle & 7 Prozent \\
\hline
\end{tabular}

Tabelle 2: Die Fälle im Programm »Familie im Stadtteil « bestehen zum größten Teil aus »Normalfamilien«: Eltern mit ihren Kindern.

\begin{tabular}{|l|l|l|}
\hline $\begin{array}{l}\text { Familien mit einem Kind oder } \\
\text { mit zwei Kindern }\end{array}$ & 70 Fälle & 62 Prozent \\
\hline Familien mit drei Kindern und mehr & 43 Fälle & 38 Prozent \\
\hline
\end{tabular}

Tabelle 3: Die Familien im Programm »Familie im Stadtteil « sind überdurchschnittlich groß.

\begin{tabular}{|l|l|l|}
\hline Unter 20 Jahre & 3 Fälle & 3 Prozent \\
\hline 20 bis unter 27 Jahre & 11 Fälle & 10 Prozent \\
\hline 27 bis unter 31 Jahre & 32 Fälle & 28 Prozent \\
\hline 31 Jahre und älter & 67 Fälle & 59 Prozent \\
\hline
\end{tabular}

Tabelle 4: Das Alter der Mütter im Programm »Familie im Stadtteil « liegt höher als erwartet. 


\begin{tabular}{|l|l|l|l|l|l|l|}
\hline Herkunftsländer & \multicolumn{2}{|c|}{$2006 / 2007$} & \multicolumn{3}{c|}{$2007 / 2008$} & \multicolumn{3}{c|}{$2008 / 2009$} \\
\hline Türkei & 27 Fälle & 37,5 Prozent & 38 Fälle & 40 Prozent & 45 Fälle & 40 Prozent \\
\hline Deutschland & 31 Fälle & 43 Prozent & 31 Fälle & 32 Prozent & 33 Fälle & 29 Prozent \\
\hline Osteuropa & 9 Fälle & 12,5 Prozent & 17 Fälle & 18 Prozent & 20 Fälle & 18 Prozent \\
\hline Herkunft binational & 0 Fälle & 0 Prozent & 7 Fälle & 7 Prozent & 11 Fälle & 10 Prozent \\
\hline Afrika, Asien & 0 Fälle & 0 Prozent & 3 Fälle & 3 Prozent & 4 Fälle & 3 Prozent \\
\hline Westeuropa & 5 Fälle & 7 Prozent & 0 Fälle & 0 Prozent & 0 Fälle & 0 Prozent \\
\hline
\end{tabular}

Tabelle 5: Die Statistik belegt den Migrationshintergrund vielen Familien im Programm »Familie im Stadtteil«.

Die Verteilung Familiengröße (vgl. Tabelle 3) im Programm »Familie im Stadtteil « lässt sich damit erklären, dass Familien mit mehreren Kindern überdurchschnittlich Belastungen ausgesetzt sind und Hilfen brauchen, wie das Programm sie anbietet. In den FiS-Familien waren 296 Kinder. Es handelt sich überwiegend um Säuglinge, Kleinkinder und Kinder im Kita-Alter (153) und im Alter der Klassen 1 bis 4 (91). 31 Familien hatten Kinder im Sekundarschulalter und 21 Familien hatten bereits ältere Jugendliche.

Entgegen der Annahme, dass überwiegend junge Familien entlastende Hilfen nachfragen, liegt der Schwerpunkt bei den älteren Familien (vgl. Tabelle 4). 97 von 113 Müttern sind älter als 27 Jahre, das sind 86 Prozent. Die FiS-Familie hatte durchschnittlich 2,6 Kinder.

Die Statistik der letzten drei Berichtsjahre zeigt, dass »Familie im Stadtteil «

\begin{tabular}{|l|c|c|}
\hline Wie die FiS-Assistentin konkret hilft & Fälle & Prozent \\
\hline $\begin{array}{l}\text { Zeit mit Kindern verbringen } \\
\text { (mit Kindern spielen, Kinderbetreuung) }\end{array}$ & 1.064 & 36 \\
\hline Hilfe beim Transport von Kindern zur Kita oder Schule & 488 & 17 \\
\hline Hilfe bei der Erziehung, Hausaufgabenbetreuung & 468 & 16 \\
\hline Entlastung durch Hilfe im Haushalt & 215 & 7 \\
\hline Unterstützung bei Freizeitaktivitäten & 172 & 6 \\
\hline »Rat und Tat« im Alltag & 160 & 5 \\
\hline $\begin{array}{l}\text { Soziale Kontakte (andere Eltern treffen, Freundschaften } \\
\text { schließen und pflegen) }\end{array}$ & 133 & 4 \\
\hline Hilfe und Begleitung beim Umgang mit Kita, Schule, Behörden & 95 & 3 \\
\hline Anderes & 142 & 5 \\
\hline
\end{tabular}

Tabelle 6: Die konkreten Unterstützungsleistungen aus dem Programm »Familie im Stadtteil « bezogen sich vielfach auf die Betreuung der Kinder. überwiegend von Einwandererfamilien in Anspruch genommen wird. Der Anteil von Familien ohne Migrationshintergrund ist von 43 Prozent (2007) über 40 Prozent (2008) auf 29 Prozent (2009) zurückgegangen. Der Anteil von Familien aus den klassischen Migrationsländern (Türkei, Osteuropa, Afrika, Asien) hat deutlich zugenommen. Im Jahre 2007 waren es die Hälfte, die aus diesen Ländern kamen (vgl. Tabelle 5).

Die Entwicklung stützt unsere Modellthese, dass Familien aus den klassischen Migrationsländern mit größeren soziokulturellen, religiösen und ethnischen kulturellen Unterschieden zur Gesellschaft des Einwandererlandes einen größeren Bedarf an stress- und isolationsmindernden Hilfen haben und deshalb »Familie im Stadtteil « in Anspruch nehmen. Nachweislich fördert "Familie im Stadtteil « die Integration von Migranten.

\section{Wie das Programm konkret unterstützt}

Die Monatsprotokolle der FiS-Assistentinnen verzeichnen im Berichtsjahr insgesamt 2.937 Einzelleistungen unterschiedlicher Qualitäten, Inhalte und Dauer. Mit den insgesamt fast dreitausend Einzelleistungen hat »Familie im Stadtteil « ein beachtliches Ausmaß an Hilfen entwickelt (vgl. Tabelle 6).

Mehr als ein Drittel aller Kontakte (36\%) bezog sich auf »Kinderbetreuung «, beispielsweise auf die Kinder aufpassen, wenn die Eltern mal Zeit für sich brauchen oder etwas zu erledigen haben. »Mit den Kindern spielen « ist hier subsumiert, es wurde $441 \mathrm{Mal}$ angegeben $(15 \%)$. Die Kontakte der FiS-Assistentinnen mit den Familien sind bis auf wenige Ausnahmen Hilfen, wie sie von Nachbarin zu Nachbarin gegeben werden können. Es sind also keine professionellen Hilfen. Eine Ausnahme sind die in der Kategorie »Hilfen bei Erziehung, Hausaufgabenbetreuung « genannten Kontakte $(16 \%)$, die sich überwiegend auf die Betreuung von Hausaufgaben beziehen, nur zu drei Prozent auf erzieherische Hilfen. Nur hier ist die Gefahr enthalten, dass die semiprofessionellen Helferinnen Aufgaben übernehmen, für die eine Ausbildung gebraucht würde. Die FiS-Teamleiterinnen müssen in ihrer Anleitung sicherstellen, dass »Familie im Stadtteil « als niederschwelliges, nachbarschaftliches Hilfeprogramm modellgetreu arbeitet.

Im Jahr 2009 wurden 95 Hilfeprozesse im Programm »Familie im Stadtteil « abgeschlossen. Die Unterstützung der Familien durch FiS-Assistentinnen dauert überwiegend - in fast drei Viertel aller FiS-Einsätze - bis zu einem halben Jahr. In einem knappen Viertel bis zu einem Jahr, wobei die Anzahl der Kontakte pro 


\begin{tabular}{|l|c|c|}
\hline Dauer des Einsatzes & Fälle & Prozent \\
\hline Eine Woche bis zu einem Monat & 3 & 7 \\
\hline Zwei bis sechs Monate & 69 & 73 \\
\hline Sieben bis zwölf Monate & 23 & 24 \\
\hline
\end{tabular}

Tabelle 7: Die Unterstützung im Programm »Familie im Stadtteil « dauerte in der Regel nicht länger als ein halbes Jahr.

Woche in der Regel niedrig ist. 26 Kontakte sind es pro Familie bei den insgesamt 113 Familien im Programm (vgl. Tabelle 7).

\section{Wie die Familien die Hilfeleistungen bewerten}

Die »Kundenzufriedenheit « wird in einem halbstandardisierten Abschlussinterview erhoben, das die FiS-Teamleiterinnen mit jeder Familie führen, wobei die Assistentinnen als Betroffene von Lob und Kritik nicht anwesend sind. Die gestellten Fragen werden von den Kunden beantwortet und von den FiS-Teamleiterinnen mitgeschrieben.

Die Zufriedenheit der Nutzer mit »Familie im Stadtteil« ist nach wie vor außerordentlich hoch. Von den 95 Familien, die Unterstützung durch »Familie im Stadtteil « hatten, gaben 88, das sind 93 Prozent, an, ihre vor der Inanspruchnahme bestehenden Ziele, wären mit »Familie im Stadtteil « realisiert worden. Vier Nutzer waren der Meinung, sie hätten ihre Ziele teilweise verwirklichen können. Nur zwei Familien gaben an, ihre Ziele seien nicht verwirklicht worden, eine Befragte machte keine Angaben.

Alle 95 Kundinnen empfanden die Unterstützung durch »Familie im Stadtteil « als wichtig. Die Häufigkeiten zeigen, dass »Familie im Stadtteil « inzwischen nicht nur etabliert ist und in hohem Maße von den Familien akzeptiert wird, sondern dass die Eltern, meistens Mütter, mit der Arbeit von »Familie im Stadtteil « und den FiS-Assistentinnen auch als Persönlichkeiten äußerst zufrieden sind.

Noch interessanter ist die Betrachtung der frei formulierten Kommentare, weil hier meist die spezifischen Unterstützungsleistungen durch die Familien bewertet wurden. Die folgenden Beispiele stehen für insgesamt 72 Voten (7).

Einige Kunden betonen, dass sie " $\mathrm{Fa}$ milie im Stadtteil « an andere Familien in gleicher Lage werter empfehlen würden:
- »Hat mir supergut gefallen. Würde es immer wieder in Anspruch nehmen. Habe >Familie im Stadtteil einigen Bekannten empfohlen.«

- »Das Programm war gut. FiS-Assistentin war immer zuverlässig. Kann man weiterempfehlen.

Die ausführlicheren Kommentare enthalten meist weitere Angaben über die Inhalte geleisteter Hilfen und geben Hinweise auf die Motive der Familien für die Inanspruchnahme von »Familie im Stadtteil«. Familien mussten, um Hilfen von »Familie im Stadtteil « nachzufragen mitunter zunächst die eigene Skepsis gegenüber dem Programm überwinden (8):

- »Die FiS-Assistentin hat mir sehr geholfen, sie ist liebevoll mit den Kindern umgegangen. Sie ist auf unsere Wünsche eingegangen, die Betreuung war sehr angenehmen. 'Familie im Stadtteik ist ein gutes Angebot. Zuerst war ich skeptisch und dachte, so was kommt für uns nicht infrage, aber es war für uns gut gewesen. So ein Projekt kann man nur weiter empfehlen. «

- „Das Projekt finde ich gut, eine tolle Idee. Ich habe mich zu erst nicht getraut, die Hilfe in Anspruch zu nehmen. Als ich mich getraut habe, war alles nur gut."

Sehr häufig wurde die Zufriedenheit mit »Familie im Stadtteil « mit der enormen Entlastung begründet, die insbesondere die Mütter erfuhren. Die Entlastungen bestanden oft darin, dass sich die FiSAssistentin um die Kinder kümmerte, um der Mutter Zeit zur eigenen Verfügung zu haben. Von außen betrachtet: Kleine Hilfen hatten immense Bedeutung für die Mütter. Für die Bedeutung, dass jemand mit ihnen spielte, wenn die Mutter Zeit für sich selbst brauchte, sprechen folgende Beispiele:

- »Ich hatte mehr Zeit für mich. Große Entlastung. «

- »Die FiS-Assistentin hat mir geholfen, es war gut und ich konnte in der Zeit in da war, der die Kinder beaufsichtigte und
Ruhe meine Sachen erledigen, z. B. Einkaufen, Termine wahrnehmen. Die Kinder mochten die FiS-Assistentin sehr, sie war für sie wie eine Oma. Es war richtig schön, weil unsere Kinder sehen ihre richtige Oma höchstens einmal im Jahr. «

- »Ich war mit der Betreuung sehr zufrieden. Die FiS-Assistentin hat gut meine Tochter beschäftigt, hat viele Ideen gehabt, es war nicht langweilig für sie. Ich hatte Zeit meine Termine nutzen zu können.«

- »Es war eine Entlastung für mich. Ich bin berufstätig. Ich musste mich nicht nach der Arbeit beeilen, um meine Kinder rechzeitig aus der Krippe abholen zu müssen. Ich wusste, dass die weiter in guten Händen sind. Die FiS-Assistentin hat sich gut um meine Kinder gekümmert. «

FiS bedeutete für Mütter auch, dass sie ihren eigenen Interessen nachgehen konnten, beispielsweise an Frauengruppen teilnehmen:

- »Die Betreuung war sehr gut, die FiSAssistentin war sehr kompetent und ist auf die Wünsche meiner Kinder eingegangen. In der Zeit der Betreuung konnte ich an den Frauengruppentreffen teilnehmen."

- »Ich habe die Betreuung in Rahmen einer Frauengruppe in Anspruch genommen. In der Zeit der Kinderbetreuung konnte ich ungestört an den Aktivitäten der Gruppe teilnehmen. Die FiS-Assistentin war sehr nett und sie konnte gut mit den Kindern umgehen. Die Kinder mochten sie sehr."

- »Dank der Kinderbetreuung habe ich die Prüfungen geschafft. Es war alles super, es war richtig gut. Die Hilfe hat mir gut getan. Die FiS-Assistentin war sehr flexibel, sie war sehr zuverlässig und die Kinder hatten viel Spaß mit ihr, sie konnte sie gut beschäftigen. Ich werde sicherlich immer noch Kontakte mit der FiS-Assistentin noch weiter pflegen."

- »Meine Tochter war sehr gut aufgehoben. Es war toll, eine FiS-Assistentin zu haben. Es war eine Entlastung für mich. In der Zeit der Betreuung konnte ich mich verstärkt um die Zwillinge kümmern."

- »Ich habe ein Kind, das in seiner Entwicklung verzögert ist und sehr viel Aufmerksamkeit braucht. Sein Bruder bekam durch die FiS-Assistentin mehr 
Zuwendung und das hat ihm viel gebracht. Es war eine große Entlastung, weil ich in der Zeit zweimal krank war."

- »Sie war sehr geduldig und unser Sohn war sehr zufrieden. Schade, dass es nur sechs Monate gedauert hat. Im nächsten Schuljahr wird er Russisch in der Schule haben, die Grundkenntnisse hat er schon."

- »Wir sind nach Bremerhaven umgezogen und wir wussten nicht, wo wir unseren Kinder Freizeit organisieren können. Der FiS-Assistent hat meinem Sohn ermöglicht, verschiedene Vereine und Einrichtungen kennenzulernen. Ich hatte nicht so viel Zeit für meinen Sohn. >Familie im Stadtteil hat meinem Sohn geholfen, sich in Bremerhaven in dem Stadtteil gut zu orientieren. Das Projekt kann ich nur weiter empfehlen.«

Wenn Kinder eingeschult werden, entsteht sowohl für die Kinder als auch für die Eltern eine Stresssituation. Das Kind und seine Eltern werden mit einer öffentlichen Einrichtung konfrontiert. Das Einfügen in eine neue Ordnung, das Lernen neuer Verhaltensregeln und die Anforderungen, neue intellektuelle und soziale Leistungen erbringen zu müssen, überfordern nicht wenige Familien und belasten sie in besonderer Weise. Die Kommentare der Mütter weisen darauf hin, dass die Entscheidung richtig war, "Familie im Stadtteil « auf Familien mit Kindern im Grundschulalter auszuweiten:

- »Wir sind mit der FiS-Assistentin zufrieden. Unser Sohn hat sich in der Schule verbessert. Er hat sehr viel Spaß mit der FiS-Assistentin gehabt. FiS-Assistentin hat uns bei den Schulgesprächen begleitet, es war sehr hilfsreich."

- »Die FiS-Assistentin hat sich gut mit unseren Schulkindern beschäftigt. Sie hat gut mit denen Hausaufgaben gemacht. Für die Kinder war es gut und wir hatten eine Entlastung, weil wir beide keine gute Bildung haben."

- »Es war sehr gut für unsere Tochter, mit ihr wurden die Hausaufgaben gemacht. Wir können ihr nicht viel helfen, weil wir die deutsche Sprache nicht gut kennen. Wir werden $>$ Familie im Stadtteil weiter empfehlen. Die FiS-Assistentin war sehr geduldig. «

- »Der FiS-Assistent war toll, er ist ein guter Mensch. Mein Sohn mochte den sehr. In den Tagen, wo der FiS-Assi- stent kam, war mein Sohn sehr ausgeglichen und hat gern seine Hausaufgaben gemacht. Ich war sehr zufrieden. «

- »Die Hilfe war wunderbar, ohne die hätte ich Schwierigkeiten mein Kind zur Sprachtherapie zu bringen. Die FiSAssistentin war pünktlich und zuverlässig."

- »Familie im Stadtteil««war große Hilfe für mich, weil ich selbst kein Auto fahre. Durch die FiS-Assistentin hat meine Familie große Entlastung bekommen."

- »Die FiS-Assistentin war sehr nett. Sie hat mich bei Behörden begleitet, beispielsweise zur Schulanmeldung, bei der Wohnungssuche oder sie hat Telefonate erledigt. Ich verstehe nicht so gut Deutsch und das ist mein Problem."

- »Das Projekt finde ich toll, die Bindung zu meinem Kind ist mit der FiS Assistentin gewachsen. Dadurch, dass das FiS-Projekt in der Kita ist, kann man die Zielgruppe gut erreichen. «

- »Ich war froh, dass die FiS-Assistentin da war. Sie hat mich bestärkt in meinem Vorhaben. Sie hatte immer ein offenes Ohr für mich. Sie hat uns einen Weg gezeigt, wie man die Probleme bewältigen kann. Ich selbst hatte für mich zu wenig getan und vieles verdrängt. Durch die FiS-Assistentin bin ich ein anderer Mensch geworden, ich bin fröhlicher. Die FiS-Assistentin hat uns bei Schuldnerberatung begleitet. «

- "Sie hat mich und meine sehr lebendigen Kinder so akzeptiert, wie wir sind. Mit ihr konnte ich darüber reden, was gibt es für mich für Möglichkeiten, wo ich mich weiter bilden oder schulen kann. Vielleicht werde ich die Schule für Eltern machen. Die Assistentin war mal länger krank, hat aber gleich die Termine abgesagt und neue vereinbart. Deshalb kommt sie auch zu uns ein Monat länger als geplant. «

Die Voten der Mütter geben »Familie im Stadtteil« ein gutes Zeugnis für die kompetente Organisation, was auf eine qualifizierte Arbeit der FiS-Teamleiterinnen schließen lässt.

- »Ich möchte FiS-Assistentin werden, damit ich Kontakt mit Deutschen bekomme.«

- »Hat mir sehr geholfen. Möchte jetzt auch helfen und FiS-Assistentin werden.

Nicht nur bei diesen Kommentaren der Mütter im Abschlussinterview lassen sich
Parallelen zu den Motiven der FiS-Assistentinnen finden: Kontakte zu anderen Müttern, zu anderen Familien haben wollen, auch außerhalb der eigenen Familie aktiv zu sein und aus der Rolle als NurHausfrau und Mutter herauszukommen, berufliche Qualifizierung durch Bildung und Weiterbildung.

Vorgesehen ist zunächst eine halbjährige Dauer der Unterstützung durch » $\mathrm{Fa}$ milie im Stadtteil «, mit der Möglichkeit der einmaligen Verlängerung. Das entspricht unserer Absicht, dass das Programm nur bei vorübergehenden Engpässen eintreten soll und dass die Familien und die FiS-Assistentinnen danach private soziale Netze aufbauen und nutzen. $\mathrm{Zu}$ einem späteren Zeitpunkt kann »Familie im Stadtteil « jedoch wieder in Anspruch genommen werden, denn nicht alle Familien sind bereit und in der Lage, sich sozial $\mathrm{zu}$ vernetzen. Einige FiS-Kundinnen äußerten den Wunsch, dass »Familie im Stadtteil « länger dauern sollte. In diesen Fällen bedarf es besonders behutsamer Gespräche seitens der FiS-Teamleiterinnen. Den Familien wird zu Beginn des Hilfeprozesses vermittelt, dass und warum die Unterstützung durch »Familie im Stadtteil « nur für eine begrenzte Zeit vorgesehen ist.

In zwei Fällen wurde »Familie im Stadtteil « abgebrochen. In beiden Fällen wurde dies nicht dem Programm oder der FiS-Assistentin angelastet. In einem Fall wurden die Kinder während des FiS-Einsatzes vom Jugendamt aus der Familie genommen, im zweiten Fall war die junge Mutter nicht in der Lage, sich auf verbindliche Situationen einzulassen.

Neben der Entlastung der Mütter war »Familie im Stadtteil « für die Mütter wichtig, weil sich durch das Programm und die Arbeit der FiS-Assistentin neue soziale Kontakte und gemeinsame Aktivitäten ergaben. In einem Fall entstand daraus der Wunsch, selbst als FiS-Assistentin tätig zu werden, um Kontakte mit deutschstämmigen Bremerhavenern $\mathrm{zu}$ bekommen.

Über »Familie im Stadtteil « entstandene Kontakte und Freundschaften sind ein wichtiger Schritt zu einer nachhaltigen sozialen Vernetzung. Dies ist so vom Programm vorgesehen. Die FiS-Assistentinnen sind nachbarschaftliche Helferinnen auf Zeit, also keine professionellen Fachkräfte. Deshalb können und sollen sie mit den Familien, insbesondere den Müttern 
Freundschaften schließen, wenn diese sich ergeben. Dies führt zu nachhaltigen sozialen Vernetzungen.

\section{Wie das Programm Menschen vernetzt}

Das Programm »Familie im Stadtteil « hatten 17 Nutzer auch deshalb in Anspruch genommen, weil sie neue Bekannte kennenlernen wollten. Freundschaften sind aber bei 33 Familien entstanden, davon bestanden die Freundschaften bei immerhin 30 Kunden auch nach dem Abschluss noch weiter. Dieses deutliche Ergebnis spricht dafür, dass »Familie im Stadtteil « die Entstehung informeller sozialer Netze bewirkt. (9)

In Bremerhaven-Süd ist eine Kooperation mit einem Familienzentrum entstanden. Dort finden einige Aktivitäten für die FiS-Assistentinnen statt und es besteht eine gute Zusammenarbeit mit den Frühförderprogrammen - »Opstapje «, »Schritt für Schritt « oder »Hippy « -, die von der Arbeiterwohlfahrt angeboten werden. In Bremerhaven-Mitte arbeitet »Familie im Stadtteil « eng mit der Kita der Initiative Jugendhilfe Bremerhaven zusammen, in der "Familie im Stadtteil « ein eigenes Büro als Anlaufstelle unterhält. Die Leiterin der Kita sieht »Familie im Stadtteil « als eine weitere Chance für effektive Elternarbeit: » Familie im Stadtteil ist eine wunderbare Sache. «

Mitarbeitern anderer Kitas muss noch vermittelt werden, dass »Familie im Stadtteil « keine Konkurrenz, sondern eine bereichernde Ergänzung der eigenen Arbeit sein kann. "Familie im Stadtteil « arbeitet mit diversen Projekten des KitaMitte zusammen. FiS-Familien nutzen Angebote der Kita, beispielsweise die Kindersportschule. Darüber hinaus hat sich an allen drei FiS-Standorten eine produktive Zusammenarbeit mit den Hebammen entwickelt. Es besteht eine gute Vernetzung mit dem Arbeitsförderungszentrum, der "Schule für Eltern «, dem »Team Schulvermeider « und den Grundschulen. An allen Standorten ist »Familie im Stadtteil « durch Schulungen mit Sozialen Diensten, Jungentelefon, Mädchentelefon, Pro Familia und dem Dienstleistungszentrum verbunden.

Mehrere FiS-Assistentinnen wurden aus Familien des Programms gewonnen; dadurch besteht weiterhin Kontakt bei
Schulungen und anderen Treffen. Die FiS-Assistentinnen haben auch Freizeitkontakte untereinander. Im Bremerhaven-Süd frühstücken die FiS-Assistentinnen einmal im Monat zusammen. In Bremerhaven-Mitte hat sich eine Gruppe von alleinerziehenden Frauen aus FiS-Familien gebildet, die sich regelmäßig wöchentlich trifft. Ein Drittel der Familien aus dem Programm kennen sich untereinander und bleiben auch nach der Hilfe durch »Familie im Stadtteil « weiterhin in Kontakt.

Die FiS-Assistentinnen sind für die Familien, nach dem Einsatz, oft Ansprechpartnerinnen. Sie vermitteln den Familien andere unterstützende Angebote. Oft bleibt der Kontakt über die Kinder der FiS-Familien und der FiS-Assistentin bestehen.

\section{Resümee: \\ Was das Programm leistet}

Das Programm »Familie im Stadtteil « hat für die Stadt Bremerhaven einen groBen »Social Return on Investment « erbracht:

- Vom Modell zum Regelangebot: Aus dem Modellprojekt »Familie im Stadtteil « ist ein neues Jugendhilfeangebot in brauchen oder etwas zu erledigen haben. Insgesamt entsprechen die Hilfekontakte der FiS-Assistentinnen nicht nur dem Modell »Familie im Stadtteil «, sondern auch der Motivation der Freiwilligen, die mit einer nur geringen Aufwandsentschädigung ehrenamtlich arbeiten.

- »Familie im Stadtteil « fördert brachliegende Helferpotentiale: Es entspricht den theoretischen Annahmen des Modells, dass es nicht schwer war, Freiwillige zu finden, die sich beim Programm engagieren, unter geeigneten Rahmenbedingungen eine sinnvolle Arbeit erbringen und dadurch Familien helfen wollten. Die FiS-Assistentinnen konnten sich in ihrer freiwilligen Arbeit weitgehend selbst verwirklichen. Mit der Art und dem Rahmen des Angebots hat »Familie im Stadtteil « offensichtlich ein Potential an Helferinnen aktiviert, die eine Gelegenheit gesucht haben, ihre Bedürfnisse in einem angemessenen Rahmen zu befriedigen und sich für eine sinnvolle Tätigkeit zu engagieren.

- Ehrenamtliches Engagement als Lebensstil: Die Zusammensetzung und die Motive der FiS-Assistentinnen entsprechen den theoretischen Vorüberlegungen. Es überwiegen Frauen des Ty-

\section{"Als ich mich getraut habe, Hilfe anzunehmen, war alles gut «}

Bremerhaven geworden. Nach der erweiterten Stellenbesetzung wurde die in der Modellkonzeption geplante personelle Ausbaustufe der hauptamtlichen Sozialarbeiterinnen erreicht. Als Folge davon hat sich "Familie im Stadtteil « erheblich vergrößert und ist nun in allen Bereichen der Stadt präsent. Trotz des Ausbaus und enormen Wachstums hat sich »Familie im Stadtteil « modellgetreu entwickelt.

- Wachsende Nachfrage abgedeckt: Mit fast dreitausend Einzelhilfen haben die Freiwilligen eine enorme Leistung erbracht. Mehr als ein Drittel aller Kontakte bezog sich auf »Kinderbetreuung ", also mal auf die Kinder aufpassen, wenn die Eltern einige Zeit für sich pus »Neues Ehrenamtes «, die ehrenamtliche Tätigkeit als Lebensstil pflegen. (10) Die freiwillige soziale Arbeit befriedigt unterschiedliche persönliche Bedürfnisse der FiS-Assistentinnen, die eine angemessene Qualifikation erhalten. Die Arbeit mit Familien ist zeitlich und dem Aufwand nach begrenzt und wird fachlich angeleitet. Eine angemessene Aufwandsentschädigung wird gezahlt.

- Integration von Migranten: Etwa ein Drittel der FiS-Assistentinnen stammen aus Migrantenfamilien. Darunter sind Frauen aus der Türkei und arabischen Ländern. Dies gilt auch für die Familien, die das Programm »Familie im Stadtteil « beanspruchen. Die Zunahme 
der FiS-Familien aus klassischen Migrationsländern zeigt, dass Familien aus diesen Ländern mit größeren soziokulturellen, religiösen und ethnischen kulturellen Unterschieden zur Gesellschaft des Einwandererlandes einen größeren Bedarf an stress- und isolationsmindernden Hilfen haben. Es hat sich gezeigt, dass eine administrativ verordnete Mischung der Wohngebiete von deutschstämmigen und Familien mit Migrationshintergrund nicht funktioniert. (11) Die durch Organisation angestoßene informelle Vernetzung auf der Ebene der Wohnquartiere erscheint hier ein effektiverer Weg, wie »Familie im Stadtteil « zeigt.

- Stadtteilentwicklung: Die Förderung und der Aufbau von Netzwerken über ein niederschwelliges Hilfeangebot auf nachbarschaftlicher Grundlage erscheint ein guter Hebel für die Gemeinwesenentwicklung und die Integration von kulturellen und ethnischen Minderheiten mit Migrationshintergrund zu sein. Jedenfalls sprechen dafür die großen Anteile bei den Nutzern, den freiwilligen Helferinnen, den nachhaltig wirkenden integrativen Gruppen aus FiS-Familien und FiS-Assistentinnen und die Vernetzung von »Familie im Stadtteil « mit den Angeboten anderer Einrichtungen.
Nach der einjährigen Förderung durch die Europäische Kommission hat Bremerhaven »Familie im Stadtteil « im vierten Jahr finanziell und politisch getragen. Somit hat die Stadt das Verdienst, als erste Kommune ein wirklich präventives Modellprogramm zu unterhalten. Sie muss allerdings auch weiterhin Kontrollansprüche möglichst vermeiden, die das Programm gefährden können. Bei allem Verständnis dafür, dass die Stadt Verwendungsnachweise für die eingesetzten Mittel braucht, muss die Anonymität der Kunden von »Familie im Stadtteil « gesichert bleiben.

\section{Anmerkungen}

(1) Vgl. Gehrmann, G. und Müller, K. D.: Familie im Stadtteil, sozialmagazin 8/2000.

(2) Die Initiative Jugendhilfe Bremerhaven (IJB) ist ein freier Jugendhilfeträger in Bremerhaven.

(3) Gehrmann, G., Müller, K. D., Säuberlich, U.: Familie im Stadtteil, Methodenhandbuch, Regensburg und Berlin 2008 , in dem neben einem umfangreichen Methodenteil Erfahrungen des ersten Jahres eingegangen sind.
(4) So z. B. das »Home Start Programme« aus Großbritannien.

(5) Es wurden vor dem Start des Programms nicht nur die künftigen FiSTeamleiterinnen ausgebildet, sondern auch einige Mitarbeiterinnen der IJB und der Sozialen Dienste der Stadt, insgesamt elf Sozialarbeiterinnen, die später als Multiplikatorinnen dienten.

(6) Die Möglichkeit, die Vorgaben anzukreuzen, kann die Antworten beeinflussen. Deshalb sind die offen gegebenen Antworten ergänzend wichtig.

(7) Die wörtlichen Zitate drücken die Zufriedenheit der FiS-Familien aus und geben oft auch Auskunft über deren Motive für ihre Wünsche nach Unterstützung. Nicht alle Familien machten hierzu ausführliche Kommentare.

(8) Das sind nur einige ausgewählte Beispiele zur Illustration.

(9) Die Aussagen zur institutionellen und informellen Vernetzung wurden wörtlich aus dem Bericht der Team-Koordinatorin übernommen.

(10) Das neue Ehrenamt wurde von Olk untersucht und beschrieben: Olk, Th.: Sozialengagement als Lebensstil, in: Blätter der Wohlfahrtspflege 1993, S. 270 f.

(11) Gaitanides, St. Wir müssen mehr miteinander reden, Migranten und Deutsche im Stadtteil. Fachhochschulverlag, Frankfurt am Main 2006.

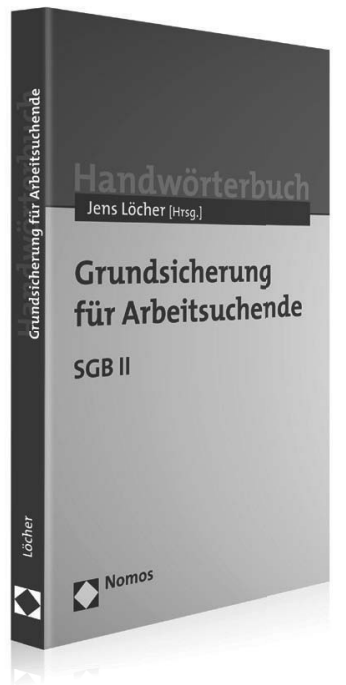

\author{
Grundsicherung für Arbeitsuchende \\ SGB II \\ Herausgegeben von Prof. Dr. Jens Löcher \\ 2011, ca. 250 S., brosch., ca. 24,- $€$, ISBN 978-3-8329-5877-O \\ Erscheint ca. August 2011 \\ nomos-shop.de/12939
}

Die Reform des Grundsicherungsrechts für Arbeitsuchende ist vollzogen. Neue Strukturen werden einbezogen, Begrifflichkeiten hinterfragt.

Das neue Handwörterbuch bietet durch Definitionen der wichtigen Begriffe - von Bildungschipkarte über Hinzuverdienstgrenze bis hin zu Zusatzleistungen bei Härtefällen - alles für den strukturierten Zugang zum reformierten SGB II. Es erläutert präzise wie verständlich das Zusammenspiel alter mit neuen Begrifflichkeiten und stellt sie in den praktischen Beratungszusammenhang. 